\title{
Comparison of L-mode regimes with enhanced confinement by impurity seeding in JET and DIII-D
}

\author{
G L Jackson ${ }^{1}$, M Murakami ${ }^{2}$, D R Baker ${ }^{1}$, R Budny $^{3}$, M Charlet ${ }^{4}$, \\ M R deBaar ${ }^{5}$, P Dumortier ${ }^{6}$, T E Evans ${ }^{1}$, R J Groebner ${ }^{1}$, N C Hawkes ${ }^{4}$, \\ D L Hillis ${ }^{2}$, L C Ingesson ${ }^{5}$, E Joffrin ${ }^{4}$, H R Koslowski ${ }^{7}$, K D Lawson ${ }^{4}$, \\ G Maddison ${ }^{4}$, G R McKee ${ }^{8}$, A M Messiaen ${ }^{6}$, P Monier-Garbet ${ }^{9}$, \\ M F F Nave ${ }^{10}$, J Ongena ${ }^{6}$, J Rapp ${ }^{7}$, F Sartori ${ }^{4}$, G M Staebler ${ }^{1}$, M Stamp ${ }^{4}$,

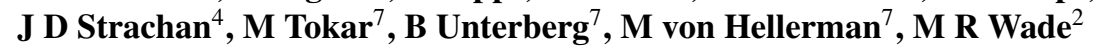 \\ and contributors to the EFDA-JET Work Programme
}

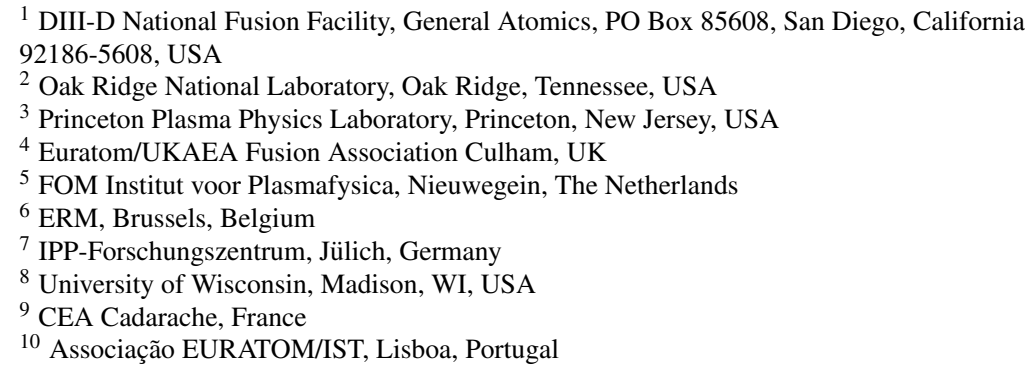

Received 16 January 2002

Published 27 August 2002

Online at stacks.iop.org/PPCF/44/1893

\begin{abstract}
Impurity seeding in both the Joint European Torus (JET) and DIII-D tokamaks has produced L-mode discharges with confinement enhancements comparable to H-mode and a near doubling of the core ion temperature when compared to similar unseeded discharges. Although $Z_{\text {eff }}$ increases with the neon injection, the total neutron rate is as high, or higher, than reference discharges and the calculated thermal neutron rate increases dramatically in both devices. Modelling with the gyrokinetic simulation code shows a reduction in low $k$ turbulence growth rates with neon injection decreasing to less than the $\boldsymbol{E} \times \boldsymbol{B}$ shearing rate, consistent with stabilization of ion temperature gradient modes in both JET and DIII-D. Reductions in ion thermal diffusivity are also observed with impurity seeding. Neoclassical $m / n=3 / 2$ tearing modes limit the duration of best performance in DIII-D with neon injection, while $n=1$ and $n=2$ magnetohydrodynamic modes limit the performance in JET.
\end{abstract}

\section{Introduction}

The use of non-intrinsic impurities in tokamaks can reduce peak heat fluxes to divertor components by reducing power flow across the last closed flux surface, which is desirable 
for fusion reactors. This reduced heat flow can also allow the discharge to remain below the $\mathrm{L}-\mathrm{H}$ power threshold, further reducing transient heat pulses, i.e. edge localized modes (ELMs), to plasma facing surfaces. An additional benefit of impurity seeding is that, under some conditions, enhanced confinement is also observed [1,2]. Neon has been injected into Joint European Torus (JET) and DIII-D discharges, increasing confinement up to $H_{97 y}=1.1$, i.e. equivalent to ELMing H-mode, while maintaining an L-mode edge. The purpose of this paper is to examine the similarities and differences in such discharges in both tokamaks, providing size scaling and leading to an understanding of the underlying physical mechanisms.

\section{Discharge evolution}

The temporal evolution of JET and DIII-D neon seeded L-mode discharges is quite similar, and is depicted in figures 1 and 2. Note that in the JET timing system, plasma current initiation begins at $t=40 \mathrm{~s}$. In this paper, we will refer to the JET discharges using this timing. In figure 1 neon is injected during the current ramp and $q_{95}$ reaches $\sim 4.1$ (JET) and 3.7 (DIII-D) at current flattop (figure $1(d)$ ). Both tokamaks exhibit a doubling of the core ion temperature (figure 1 $(a)$ ) when compared to reference (no neon) discharges. In both devices, the radiated power fraction increases to 0.5 or more with neon seeding (figure $1(b)$ ) and confinement is at or above the $H_{97 y}$ ELMing $\mathrm{H}$-mode value (figure $1(c)$ ). Thermal stored energy is used in

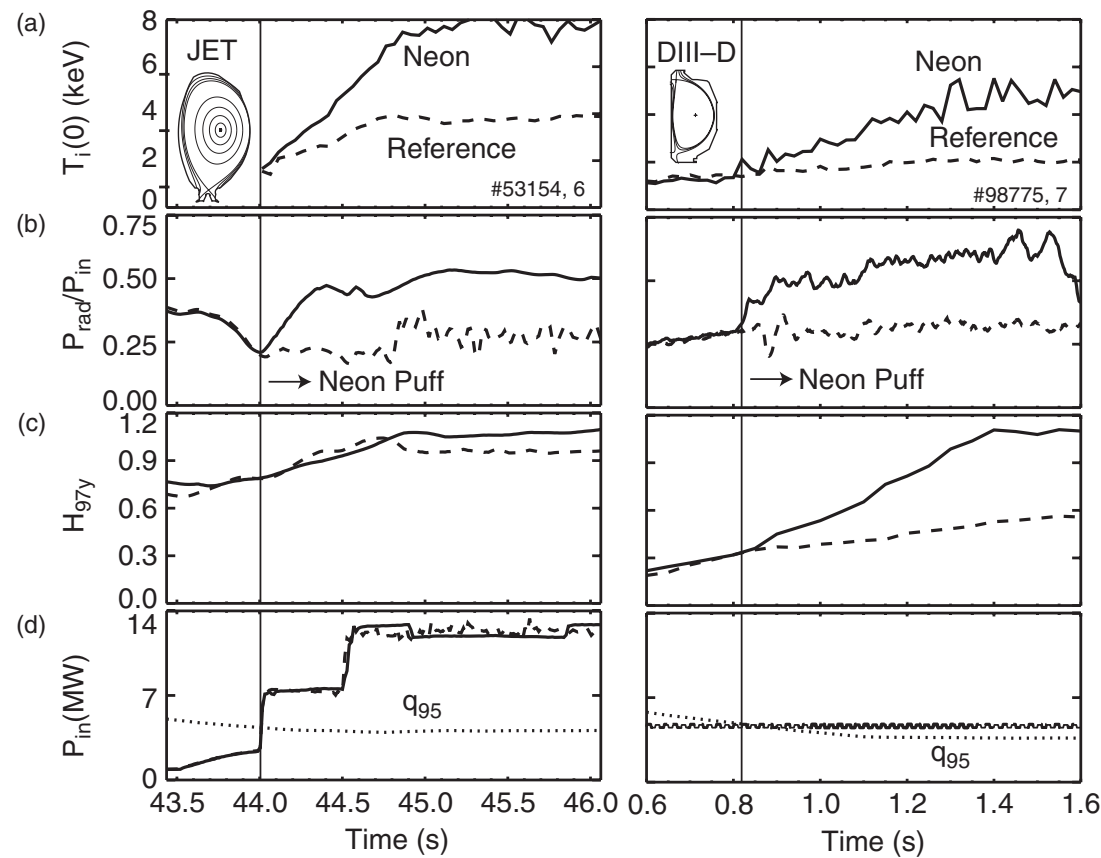

Figure 1. Temporal evolution of JET (left panels) and DIII-D (right panels) neon seeded discharges (\#53154 and \#98775). Reference discharges are also shown (- - - - \#53156 and \#98777). Plotted are: $(a) T_{\mathrm{i}}(0),(b)$ radiated power fraction, $(c) H_{97 y}$ confinement enhancement factor and $(d)$ input power. Safety factor, $q_{95}$, is also shown in $(d)$ as dotted lines. Ordinate scales are the same for both DIII-D and JET to allow a direct comparison. Discharge parameters are: (JET) $I_{\mathrm{p}}=1.7 \mathrm{MA}$, $B_{\mathrm{t}}=2.2 \mathrm{~T}, q_{95}=4.1$; (DIII-D) $I_{\mathrm{p}}=1.2 \mathrm{MA}, B_{\mathrm{t}}=1.6 \mathrm{~T}, q_{95}=3.7$. 

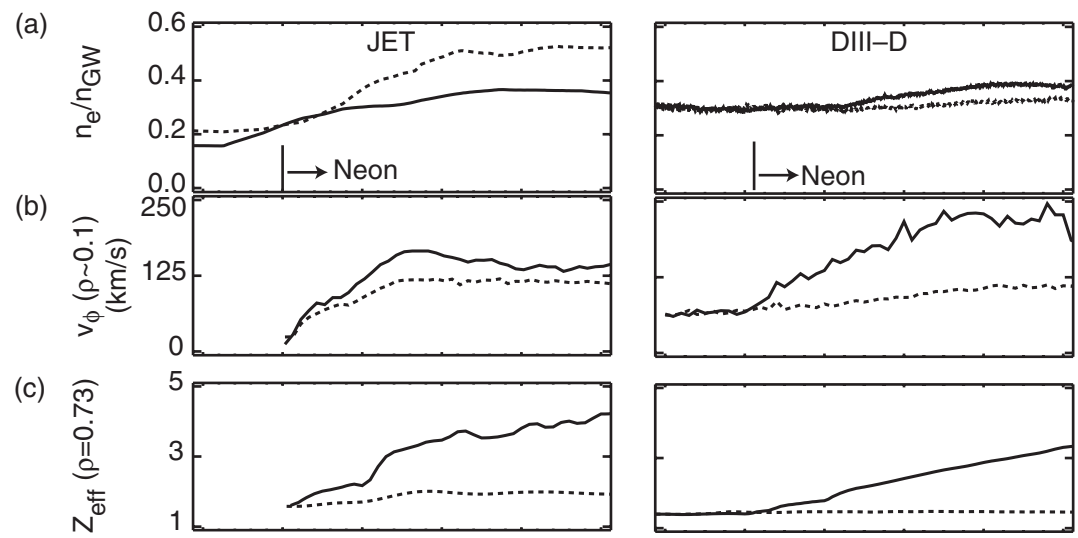

(d)

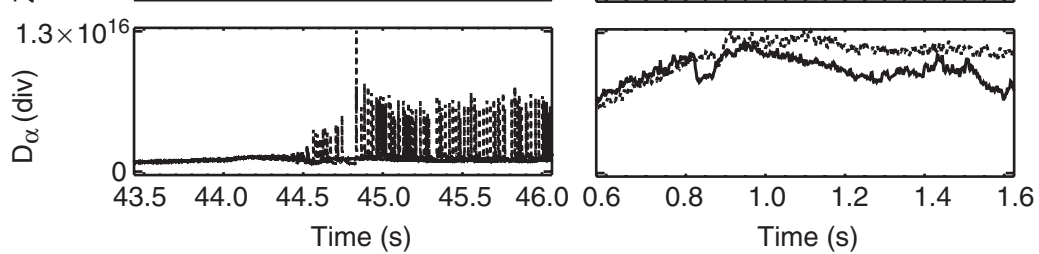

Figure 2. Plotted are (a) normalized density, $(b)$ core toroidal rotation, $(c)$ mantle $Z_{\text {eff }}$ and $(d)$ divertor deuterium line intensity $\left(\mathrm{D}_{\alpha}\right)$ for the same discharges as in figure 1. Left panels are JET discharges and right are DIII-D. Reference (no neon) discharges are shown as dashed lines.

the calculation of $H_{97 y}$, i.e. there is no contribution from fast ions in evaluating confinement in figure 1(c) for either device. Both DIII-D discharges shown in figure 1 had an L-mode edge. Note, however, that in the case of JET the reference discharge had an L-H transition at $44.45 \mathrm{~s}$, shown in figure $2(d)$, while the neon seeded discharge remained in L-mode. This difference is due to the higher mantle radiation with neon which reduces power flow across the last closed flux surface (LCFS) to a value below the H-mode power threshold in JET. In DIII-D the $\nabla \boldsymbol{B} \times \boldsymbol{B}$ drift direction is away from the X-point and the higher L-H power threshold allows the unseeded discharge to remain in L-mode. Other temporal characteristics of DIII-D and JET discharges are displayed in figure 2. The fraction of Greenwald density [3], $f_{\mathrm{GW}}=\bar{n}_{\mathrm{e}} / n_{\mathrm{GW}}$, is similar for both DIII-D and JET with neon injection (figure $2(a)$ ). In the JET reference case, the higher density is caused by the higher particle confinement time in H-mode. Both DIII-D and JET exhibit higher core toroidal rotation with neon injection, plotted in figure $2(b)$, and this increase begins promptly after neon injection. $Z_{\mathrm{eff}}$ in the mantle region is comparable in both devices, figure $2(c)$. For comparison purposes, the $Z_{\text {eff }}$ calculations use impurity line emission from charge exchange recombination to calculate impurity density profiles of neon and carbon (DIII-D) and neon, carbon, helium, beryllium and nitrogen (JET). In both machines, carbon and neon are the dominant impurities for these types of discharges.

Even though $Z_{\text {eff }}$ is higher with neon seeding, the measured neutron rate in both machines (figure 3(a)) increases with neon seeding compared to reference discharges, although the increase is not as large in JET since it is compared to a reference ELMing H-mode. There is an even more dramatic increase in the calculated thermal neutron rate with neon seeding in both devices, shown in figure $3(b)$. Calculations of the total neutron rate, $S_{\mathrm{n}}$, are compared to the rate calculated by the TRANSP code [4] in figure 3(a). The calculated neutron rate uses $Z_{\text {eff }}$ derived from charge exchange measurements, which overestimates $S_{\mathrm{n}}$ by up to $30 \%$ in JET. 


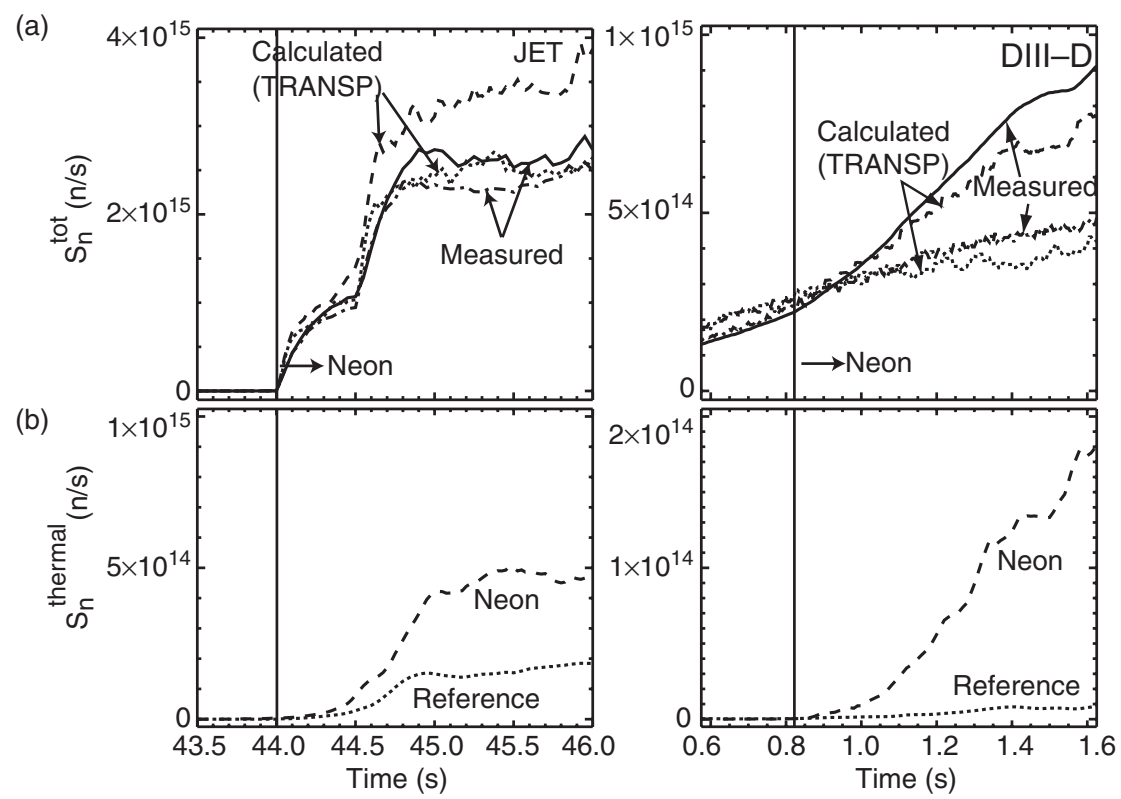

Figure 3. ( $a$ ) Total neutron rate and $(b)$ thermal neutron rate, for JET (left panels) and DIII-D (right panels). Measured neutron rate is shown as a solid line (neon) and a dash-dot line (reference). Neutron rates calculated by TRANSP are indicated by a dashed line (neon) and a dotted line (reference).

TRANSP analysis of the same JET discharge using $Z_{\text {eff }}$ measured by the visible bremsstrahlung array exhibited an even larger discrepancy, reducing the neutron rate by approximately a factor of 2 below the measured rate. For this paper, TRANSP calculations will use $Z_{\text {eff }}$ inferred from charge exchange recombination spectroscopy for both JET and DIII-D.

Profiles of the discharges in figures 1-3 are shown in figure 4 during times when effects of neon seeding are well established: $44.8 \mathrm{~s}$ (JET) and $1.16 \mathrm{~s}$ (DIII-D). Profile data is only plotted for $\rho<0.8$ due to large uncertainties in the calculation of thermal diffusivities near the LCFS. For the neon seeded discharges, with an L-mode edge in both JET and DIII-D, there is an increase in the ion temperature gradient, $-\mathrm{d} T_{\mathrm{i}} / \mathrm{d} \rho$, at $\rho \sim 0.6$ (DIII-D) and $\sim 0.35$ (JET) and extending inwards. This occurs in the region where the ion thermal diffusivity, $\chi_{i}$, is decreasing rapidly in JET (figure $4(c)$ ). Even though the reference JET discharge is in H-mode, $\chi_{i}$ with neon is lower than the reference discharge in the core, $\rho<0.3$.

In DIII-D, $\chi_{i}$ with neon seeding is at or below $\chi_{i}$ in the reference discharge at the time shown in figure 4 . While $\chi_{i}$ has been dramatically reduced over most of the profile, there is also a $50 \%$ decrease from $\rho \sim 0.7$ to $\rho \sim 0.55$ with neon (figure 4 ), which is in the region where the ion temperature gradient is increasing. Within the region of higher temperature gradient, described above, plasma thermal pressure, $n_{\mathrm{e}} T_{\mathrm{e}}+\sum n_{\mathrm{i}} T_{\mathrm{i}}$ (figure $4(b)$ ), is generally higher than the reference discharge, consistent with the lower transport in the neon seeded discharges.

\section{Physical mechanisms}

The temporal aspects of JET and DIII-D impurity seeded L-mode discharges are similar, suggesting that the underlying physical mechanisms may also be the same. In this section we first discuss DIII-D discharges and then JET. 

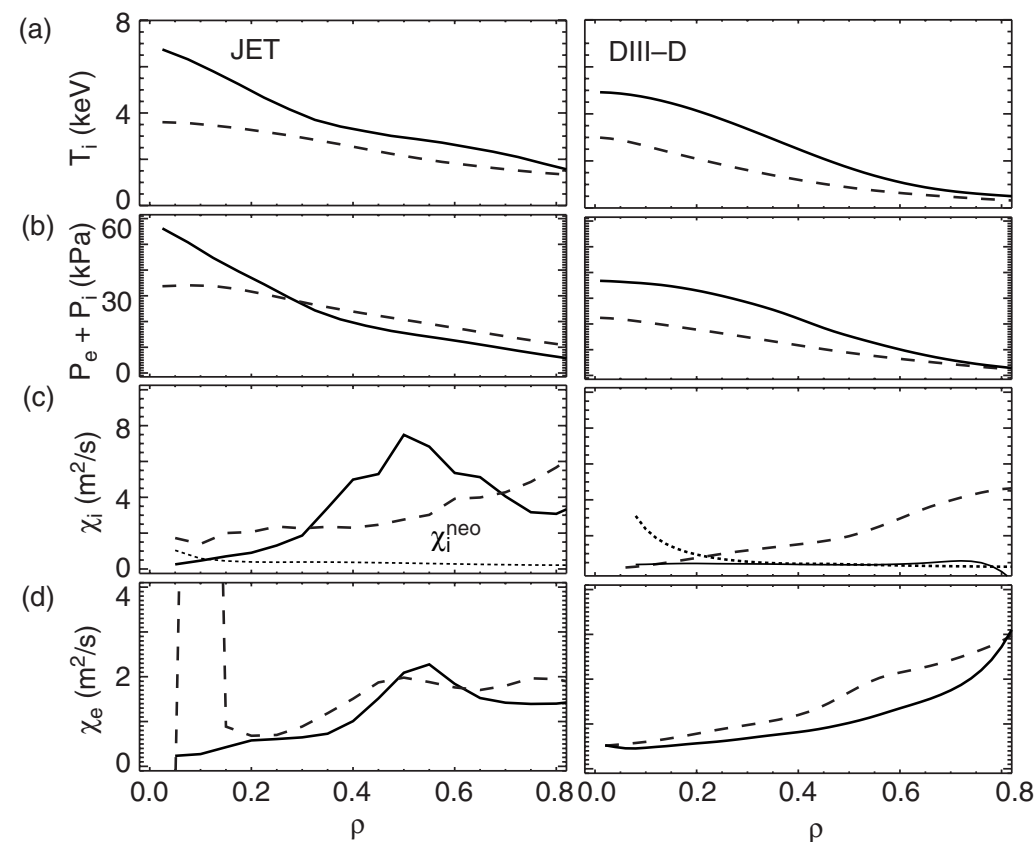

Figure 4. Profiles of $(a) T_{\mathrm{i}}$, (b) plasma thermal pressure, $(c) \chi_{\mathrm{i}}$ and $(d) \chi_{\mathrm{e}}$ for both JET (left panels) and DIII-D (right panels) at $44.8 \mathrm{~s}$ (JET) and $1.16 \mathrm{~s}$ (DIII-D). Ion neoclassical diffusivity is shown as a dotted line $(c)$. Reference discharges (no neon) are shown as dashed lines. Discharges are the same as those in figure 1.

As discussed in [5], there is a reduction of density fluctuations in DIII-D after the onset of neon injection which is measured by beam emission spectroscopy (BES) and far infrared (FIR) scattering. This reduction is most pronounced in the region $\rho \sim 0.6-0.7$ [5]. The gyrokinetic simulation (GKS) code [6] shows that the growth rates of micro-turbulence in the wavelength range of ion temperature gradient (ITG) modes are significantly reduced in this region. Recent work has shown that reductions in the growth rate of ITG micro-turbulence are possible when $\omega_{\boldsymbol{E} \times \boldsymbol{B}}>\gamma_{\max }$ where $\omega_{\boldsymbol{E} \times \boldsymbol{B}}$ is the $\boldsymbol{E} \times \boldsymbol{B}$ shear stabilization rate and $\gamma_{\max }$ is the maximum growth rate of low $k$ micro-turbulence, $k<3 \mathrm{~cm}^{-1}$ in DIII-D, in the ion diamagnetic drift direction [7,8]. Neon seeding acts to reduce the linear growth rates calculated by the GKS code and hence can be stabilizing if the reduction is large enough that $\omega_{\boldsymbol{E} \times \boldsymbol{B}}>\gamma_{\max }$ [9]. This is discussed further in section 5 .

The $\boldsymbol{E} \times \boldsymbol{B}$ shearing rates calculated using the Hahm-Burrell formulation [10] and $\gamma_{\max }$ for similar DIII-D discharges with and without neon are plotted in figure 5. For the reference discharge, the $\boldsymbol{E} \times \boldsymbol{B}$ shearing rate is less than $\gamma_{\max }$ for $\rho \gtrsim 0.55$, and shear stabilization of ITG modes would not be expected in this region. Note that the regions where $\gamma_{\max }$ is not displayed are where the GKS code could not calculate a maximum growth rate. However, with neon $\gamma_{\max }$ is well below $\omega_{\boldsymbol{E} \times \boldsymbol{B}}$ and $\boldsymbol{E} \times \boldsymbol{B}$ shear stabilization can reduce the growth rates of ITG micro-turbulence. This is consistent with the experimental observations of reduced low $k$ turbulence discussed in [5]. The calculated $k$ spectra at $\rho=0.64$ for these DIII-D discharges are shown in figure 6 . The ordinate is plotted as $\gamma / k$ in order to display both the low $k$ and high $k$ regions. For $k_{y}<3$, the turbulence phase velocities are in the ion diamagnetic drift direction, where turbulence suppression has been observed for the neon seeded discharge. Higher values of $k_{y}$ in figure 6 , greater than $3 \mathrm{~cm}^{-1}$, have phase velocities in the electron drift direction, which 


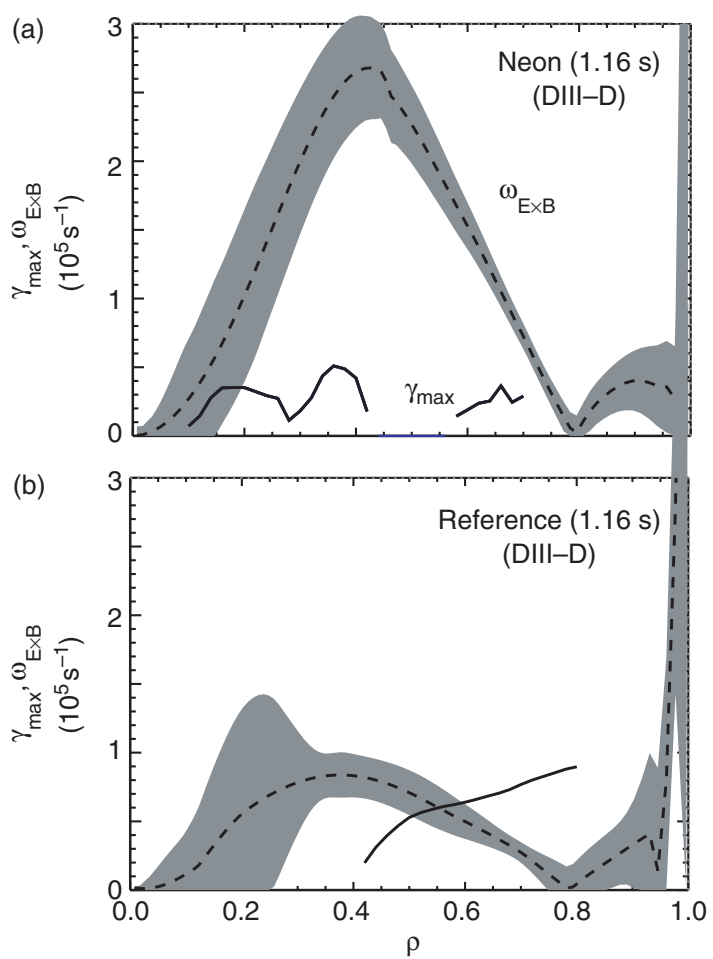

Figure 5. Maximum growth rates and $\boldsymbol{E} \times \boldsymbol{B}$ shearing rates for DIII-D discharges with $(a)$ and without $(b)$ neon seeding at $1.16 \mathrm{~s}$. DIII-D discharges are the same as in figures 1 and 2 .

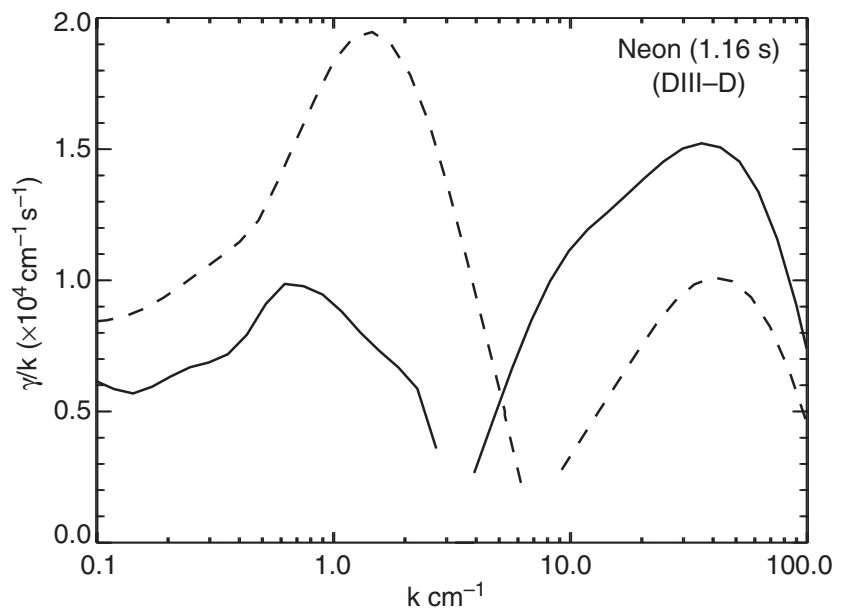

Figure 6. GKS calculated growth rates normalized to $k$ as a function of wavenumber for a DIII-D seeded discharge (\#98775,__ ) and a reference discharge $\left(\# 98777,--_{--}\right)$at $t=1.16 \mathrm{~s}$ and $\rho=0.64$.

could indicate either transverse electron (TE) or electron temperature gradient (ETG) mode turbulence. Although reductions in ETG turbulence have been observed in previous DIII-D discharges [9], the role of TE and ETG turbulence in the type of DIII-D discharges described in this paper has not been thoroughly investigated. 
(a)

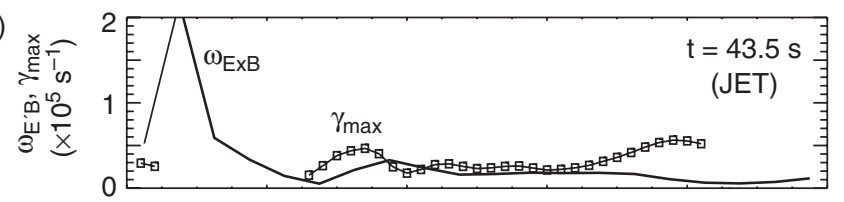

(b)

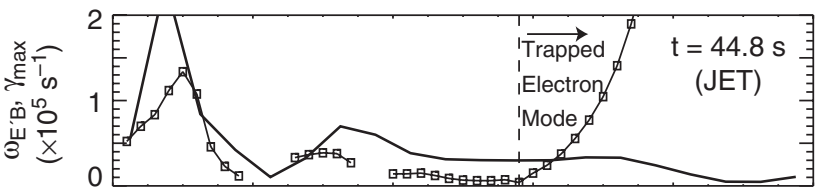

(c)

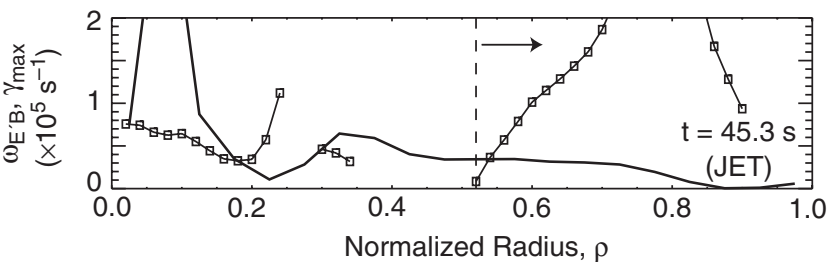

Figure 7. Maximum growth rate and calculated $\boldsymbol{E} \times \boldsymbol{B}$ shearing rate for JET pulse \#53154 with neon seeding at three times: (a) $43.5 \mathrm{~s},(b) 44.8 \mathrm{~s}$ and (c) $45.3 \mathrm{~s}$. Vertical dashed lines in $(b)$ and (c) indicated radius where the maximum growth rate is in the electron diamagnetic drift direction and higher $k$, indicating that electron effects are dominant.

GKS analysis of a JET discharge at 3 times is plotted in figure 7. Because no poloidal velocity measurements are presently available in JET, neoclassical estimates of the poloidal rotation velocity are used to calculate the $\boldsymbol{E} \times \boldsymbol{B}$ shearing rate. Since the reference JET case was an H-mode discharge, stable to ITG modes, this reference discharge is not shown. Just after neon injection (figure $7(a)), \gamma_{\max }$ and the shearing rate, $\omega_{\boldsymbol{E} \times \boldsymbol{B}}$, are comparable over most of the profile. At the time of peak core toroidal rotation, $t \sim 44.8 \mathrm{~s}, \omega_{\boldsymbol{E} \times \boldsymbol{B}}$ is stabilizing out to $\rho \sim 0.6$ (figure $7(b)$ ), beyond which the frequency of the maximum growth rate is in the electron diamagnetic drift direction where high $k$ electron micro-turbulence is dominant. At a later time (figure $7(c)$ ), when stored energy has reached a steady state, the region of electron dominated turbulence has moved inward, and there is an inward region $0.2<\rho<0.3$ where $\omega_{\boldsymbol{E} \times \boldsymbol{B}}$ is not stabilizing to low $k$ ITG modes. This is consistent with a reduction in core toroidal rotation (figure $2(b)$ ).

The $k$ spectra at $\rho=0.3$ and 0.64 for the JET neon seeded discharge displayed in figures $1-4$ is plotted in figure 8. In the core (figure $8(a)$ ), $\gamma$ is a maximum at $k$ of $\sim 1$ similar to DIII-D (figure 6), although $\gamma$ is somewhat larger than in DIII-D. This may be because the shearing rate, $\omega_{\boldsymbol{E} \times \boldsymbol{B}}$, is only slightly larger than $\gamma$ (figure $7(b)$ ) and thus the shear is only marginally stabilizing. However in the mantle region (figure $8(b)$ ), the maximum $\gamma$ occurs at $k>30$, and the frequency is in the electron diamagnetic drift direction. This is consistent with the dominant modes being electron modes producing the maximum turbulent growth rates in this region. Impurity seeding is not effective in reducing the electron induced turbulence.

\section{Effects of impurity seeding on magnetohydrodynamic modes}

Impurity seeded L-mode discharges in both JET and DIII-D are limited by magnetohydrodynamic (MHD) activity. In DIII-D $m / n=3 / 2$ neoclassical tearing modes 


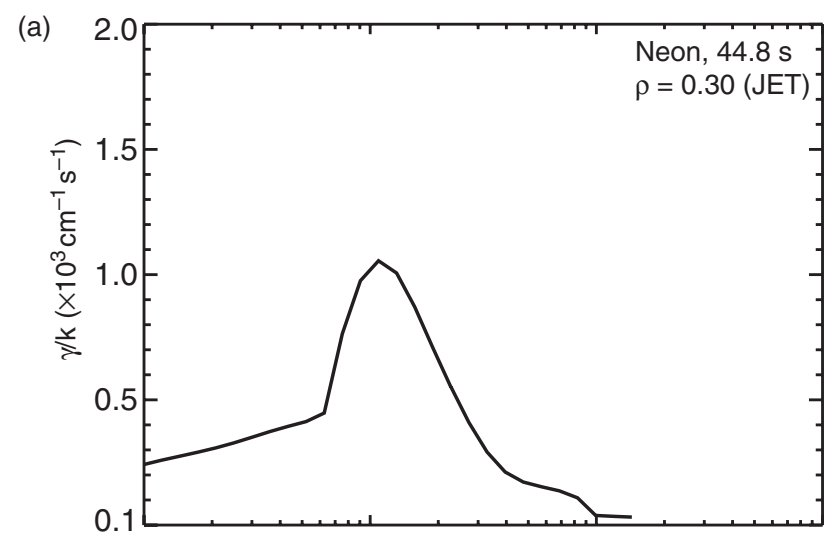

(b)

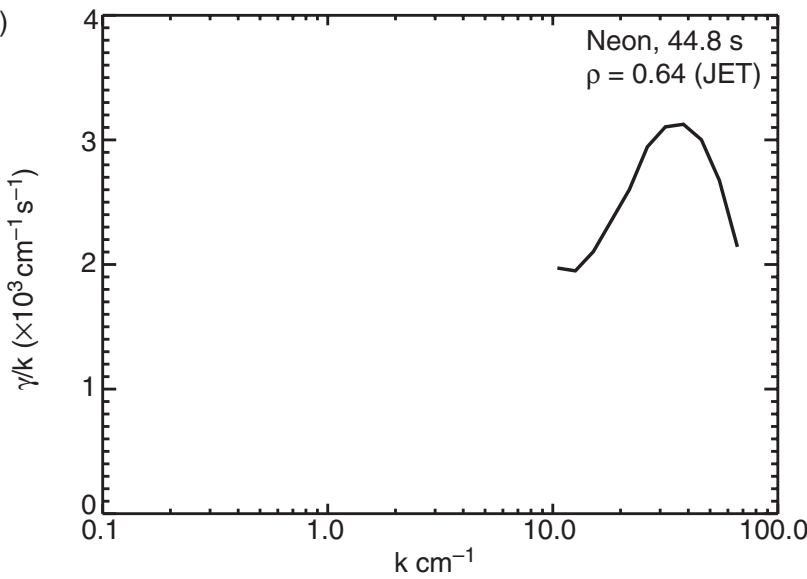

Figure 8. GKS calculated growth rates for JET neon seeded discharge \#53154 and two radii: (a) $\rho=0.30$ and $(b) \rho=0.64$ at $t=44.8 \mathrm{~s}$.

(NTMs), triggered by sawteeth, limit the duration of the enhanced performance phase. A typical example is shown in figure 9. In this example an $m / n=1 / 1$ mode begins (figure $9(d)$ ) as $q_{\text {min }}$ approaches unity followed by sawteeth (figure $9(c)$ ). Both $W_{\text {MHD }}$ (figure $9(a)$ ) and the neutron rate (figure $9(b)$ ) begin decreasing. At $t=1.88 \mathrm{~s}$, an $m / n=3 / 2 \mathrm{NTM}$ further reduces confinement and the neutron rate.

In JET, early tearing modes (not neoclassical) near the time of neon injection can severely limit performance. In addition, the lower L-H power threshold can produce edge MHD (ELMs) which is also detrimental. In other JET discharges, e.g. figure 1, higher stored energy and neutron rates may be clamped by the onset of fishbones and sawteeth. However, lower hybrid current drive (LHCD) may ameliorate the effects of $n=1$ activity. As shown in figure 10, increasing the LHCD power reduces the $n=1$ sawteeth precursors (figure $10(b)$ ). However, in this case, the $n=2$ MHD activity was not affected (figure 10(c)); therefore, additional methods are needed to reduce this tearing mode.

In both devices, MHD avoidance is a crucial next step for extending the duration of these types of discharges and evaluating their efficacy. For neon seeded L-mode discharges, electron cyclotron current drive (ECCD) suppression of the $m / n=3 / 2$ NTMs has been proposed in DIII-D [11] and further LHCD experiments may extend the duration of the high performance neon seeded discharges in JET. 
(a)

(b)
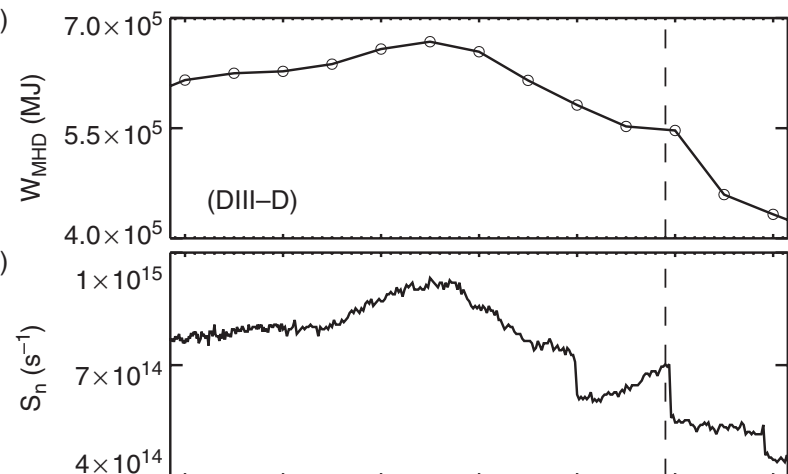

(c)

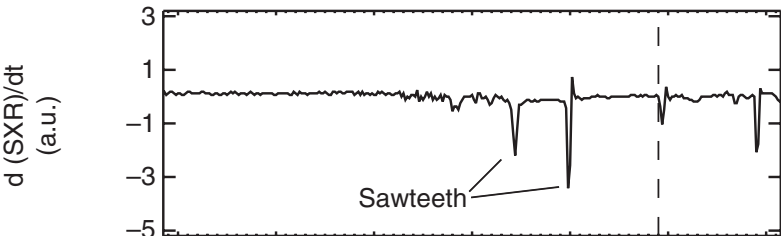

(d)

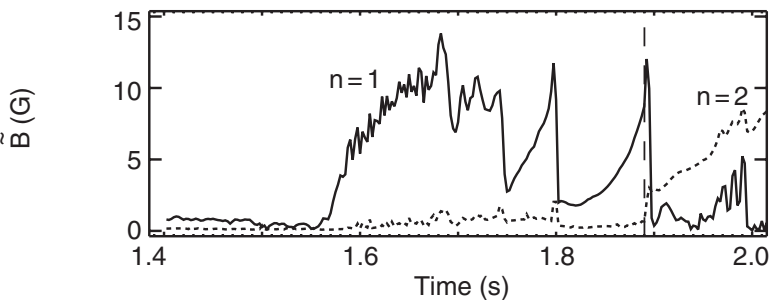

Figure 9. (a) Stored energy and $(b)$ the neutron rate both decrease after $(c)$ the onset of sawteeth and $(d) n=1$ MHD activity and further decrease after the onset of an $m / n=3 / 2$ NTM (- - - ) for a DIII-D neon seeded discharge (\#98775).

(a)

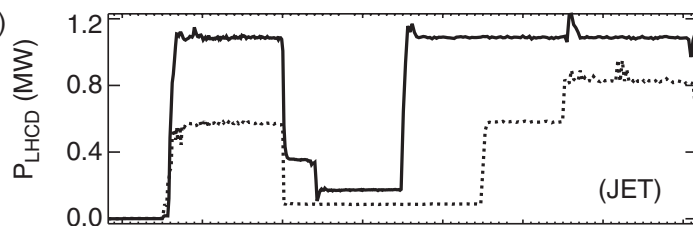

(b)

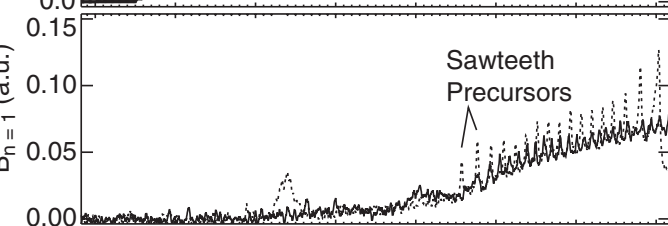

(c)

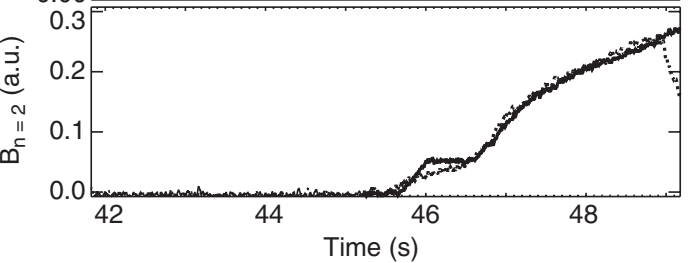

Figure 10. Plotted are: (a) LHCD power, $(b) n=1$ MHD amplitude and $(c) n=2$ MHD amplitude. The excursions in $B_{n=1}$ are sawteeth precursors. Both neon seeded discharges $(\# 52180,52183)$ have similar parameters. 


\section{Conclusions}

Neon seeded discharges with an L-mode edge and confinement equivalent to ELMy H-mode have been achieved in both DIII-D and JET. The temporal evolution of these discharges is similar in both devices. When compared to reference (unseeded) discharges, a region of higher ion temperature is observed, up to a factor of 2 in the centre. Higher plasma pressure extends from the core to $\rho \sim 0.6$ in DIII-D and $\rho \sim 0.35$ in JET. The smaller region in JET is one of the differences between these two tokamaks. Whether this is due to differences in operation or is a fundamental difference has not been established.

In both devices, $\boldsymbol{E} \times \boldsymbol{B}$ shear stabilization of low $k$ (ITG) modes appears to be the physical mechanism leading to lower thermal diffusivities and higher confinement. BES measurements in DIII-D show a reduction in density fluctuations in the wavelength range where such microturbulence is expected. Although such measurements are not available in JET, $\boldsymbol{E} \times \boldsymbol{B}$ shear stabilization in the region $\rho \lesssim 0.35$ can account for the lower diffusivity and higher ion temperatures. A model which is consistent with these observations is that impurity seeding acts synergistically to reduce low $k$ ion turbulence (ITG modes). Initially the introduction of neon acts as a trigger to decrease the growth rate of the turbulence by increasing the impurity concentration (mass stabilization). This reduction of turbulence leads to lower transport and higher $\boldsymbol{E} \times \boldsymbol{B}$ shear producing additional stabilization and creating a positive feedback loop.

Future work will be directed at extending the duration of these neon seeded discharges by reducing MHD, using tools such as ECCD and LHCD.

\section{Acknowledgments}

This paper is partially supported by the US Department of Energy under Contract Nos DE-AC03-99ER54463, DE-AC05-00OR22725 and DE-AC02-76CH03073, and Grant No DE-FG03-96ER54373.

\section{References}

[1] Ongena J et al 1999 Plasma Phys. Control. Fusion 41 A379

[2] Murakami M et al 2000 Nucl. Fusion 41317

[3] Greenwald M, Terry J L, Wolfe S M and Ejima S 1988 Nucl. Fusion 282199

[4] Goldston R J et al 1981 J. Comput. Phys. 4361

[5] McKee G R et al 2000 Phys. Plasmas 71870

[6] Waltz R E and Miller R L 1999 Phys. Plasmas 64625

[7] Burrell K H 1997 Phys. Plasmas 41499

[8] Waltz R E et al 1994 Phys. Plasmas 12229

[9] Staebler G M et al 1999 Phys. Rev. Lett. 821692

[10] Hahm T S and Burrell K H 1995 Phys. Plasmas 21648

[11] LaHaye R J 2002 Control of neoclassical tearing modes in DIII-D, Presented at the 43rd Meeting of the American Physical Society, Division of Plasma Physics (Long Beach, California, USA) Phys. Plasma B at press 